\title{
Vortex ropes in draft tube of a laboratory Kaplan hydroturbine at low load: An experimental and LES scrutiny of RANS and DES computational models
}

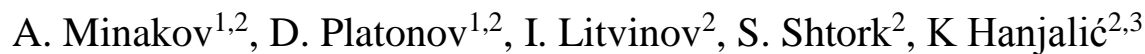 \\ ${ }^{I}$ Siberian Federal University, Krasnoyarsk, Russia, tov-andrey@yandex.ru \\ ${ }^{2}$ Novosibirsk State University, Novosibirsk, Russia, platonov-08@yandex.ru \\ ${ }^{3}$ Delft University of Technology, Chem.Eng. Dept., k.hanjalic@tudelft.nl
}

\begin{abstract}
We performed numerical simulation complemented by experiments of flow in a 60:1 scaled-down laboratory model of a Kaplan hydroturbine at a part load of about $40 \%$ nominal flowrate, using two eddyviscosity- (EVM) and a Reynolds stress (RSM) RANS models (realizable k- $\varepsilon$, k- $\omega$ SST, LRR) and detachededdy-simulations (DES), all on $2 \mathrm{M}$ (million) and $6 \mathrm{M}$ grid cells, as well as large-eddy simulations (LES) on $6 \mathrm{M}$ and 19.3M grids. Unlike the linear EVMs, the RSM, DES (on 2M grid), and LES (on 6M grid) reproduced well the mean velocity components, the $r s m$ of their fluctuations and pressure pulsations in the diffusor draft tube. Despite relatively coarse meshes and insufficient resolution of the near-wall region, LES, DES and RSM also reproduced well the intrinsic flow unsteadiness and the dominant flow structures, all capturing a twin-rope pattern and the associated pressure pulsations in the draft tube. The EVM URANS on 2M grid could not maintain unsteady solution, but did so on a finer grid of $6 \mathrm{M}$, resulting in improved mean-flow patterns, but still failing to capture the twin-helix structures and the associated pressure pulsations.
\end{abstract}

Keywords: hydroturbine draft tubes, vortex ropes, pressure pulsation, LES, RANS and DES models.

\section{Introduction}

The common hydraulic turbomachinery have long reached a mature stage of development and at design conditions they usually perform with high efficiency and reliability. However, at part loads and in transient regimes the stability of the system can seriously be impaired leading to a decrease in efficiency, mechanical damage, fatigue and system failure. The intrinsic unsteadiness at suboptimal conditions leads often to vortex breakdown and precessing helical vortices in form of a single or twin rope behind the impeller, which cause intense flow pulsations and vibrations of the turbine structure that pose serious threat to the system reliability and safety. These issues have long been in focus of the experimental research complemented by some simplified analytics, but a full account of the threedimensional time dynamics has remained to a large extent beyond the reach of even most advanced laser-based measurements and diagnostics techniques. Computer simulations have been seen as a potential tool for capturing the subtleties of the development of instabilities and associated vortical and turbulence structures. Such information can be obtained by direct and large-eddy simulations (DNS, LES) over a range of important scales. However, the accuracy of DNS and LES depends much on the numerical resolution. For complex configurations the Reynolds-averaged Navier-Stokes (RANS) approach, being computationally less demanding, has been considered as a more rational option for industrial purposes. However, because if its empirical contents, the RANS have not been regarded as a trustful research tool, and moreover, their credibility depends much on the choice of the turbulence model to close the time- or ensemble-averaged conservation equations. 
Despite the extensive literature (e.g. Doerfler [1], Alligne et al. [2], comprehensive experimental data for hydroturbines are still scarce, apart from mean velocity and secondmoment turbulence statistics measured at selected locations in laboratory models of some popular turbine types [3.4]. On the other hand, there are still many open questions regarding the numerical simulation of flows in turbines of realistic configuration and practical relevance. Because of typically very high Re numbers, DNS and even LES, requiring enormous numerical mesh and computer resources, are usually beyond the reach of designers, but also of many researchers, leaving the URANS or hybrid RANS/LES as the only viable routes. Here the first challenge is related to selecting the appropriate RANS model that can reproduce satisfactorily the unsteady flow and the dynamics of at least the dominant largescale vortex structures subjected to strong swirls, curvature and adverse pressure gradients in complex geometric shapes. The popular linear eddy-viscosity modes (k- $\varepsilon, \mathrm{k}-\omega$, and their many "improved" variants) lack on sufficient physics and have shown to perform poorly in describing such flows.

We report here on simulation of flow in a scaled-down model of Kaplan turbine at a part load using several RANS models, a DES (detached-eddy simulations) and LES. The models were benchmarked against the experimental data (partly reported by Minakov et al. 2014), as well as against the present reference LES, run on a fine grid with 19.3 million cells. We discuss the flow patterns and vortical structure in the turbine draft tube (DT) and its impact on flow stability and pressure pulsations, especially at part loads characterized by unsteady twin helix ropes.

\section{Flow configuration and the experiment}

An experimental study was performed on an open aerodynamic stand. The flow configuration considered is a 60:1 scaled-down laboratory model mimicking the draft tube of an LMZ (Leningrad Metal Industry) Kaplan turbine from a hydropower plant, operating at a low load with a volumetric flowrate of $19 \mathrm{l} / \mathrm{s}\left(68.4 \mathrm{~m}^{3} / \mathrm{h}\right.$, about $39 \%$ of the nominal capacity) corresponding to $\mathrm{Re}=89.000$, at a head of $1.67 \mathrm{~m}$ and rotating speed of $2273 \mathrm{rpm}$. The laboratory turbine actually does not look precisely like the typical Kaplan turbine, but nevertheless it was designed to generate the same inflow conditions into the draft tube as in the full scale prototype. Namely, as demonstrated in [5-7], the conditions in a real turbine setup could be achieved in an experiment without having to replicate precisely the full geometry of the complete real turbine system (spiral chamber, stay vanes and guide vanes, rotor). What matters for studying the draft tube flow dynamics is the inflow velocity and turbulence field distribution after a real turbine, entering the draft tube. These have been created using a set of two rows of blades, designed by LMZ to generate the draft tube inlet conditions that corresponds to a real Kaplan turbine. To this effect one of the rows was set still and functions as a guide vane of the turbine (with a uniform fluid inflow at a volumetric flowrate $Q$ ). The second row forcedly rotates with frequency $f$ and is an analogue to the turbine impeller. Thus, by controlling the two parameters $Q$ and $f$ it is possible to experimentally simulate the modes of the turbine generator load.

Air supply in the draft tube model was carried out using a high performance air blower

with a maximum flowrate $Q_{\max }=550 \mathrm{~m}^{3} / \mathrm{h}$ and pressure excess of $\triangle P=0.4$ bar. Air flowrate was controlled by an ultrasonic flowmeter "IRVIS-Ultra" and frequency converter of the 
blower with feedback. For capturing the LDA signal the air flow was seeded with tracers produced by a standard-type generator of the paraffin oil aerosol with a mean particle diameter of $1-3 \mu \mathrm{m}$.

Figure 1 provides a photo and a schematic of the experimental turbine model. The air flow with tracers was supplied to an axisymmetric chamber through six bends. To suppress the influence of the bends and align the airflow entering the guide vanes, two honeycombs and a profiled nozzle were used. The air flows through the cascade of blades and enters the draft tube cone. The draft tube model and a pair of blade rows "guide vanes - impeller" were provided by the company LMZ [8] and manufactured using the technology of 3D printing. Servo drive SPSh10-3410 ensured precise setting of rotation frequency of the impeller in the range from 0 to $3000 \mathrm{rpm}$. The control of the experiment was ensured by a computer. Using the original software it was possible to maintain the present flow regime with an accuracy of 1.5 and $0.5 \%$ respectively for the flowrate and the impeller rotation frequency.

The experiments included measurements of velocity fields in the conical part of the draft tube using a system of two-component LDA "LAD-06i", as well as of pressure pulsations with special acoustic sensors. The acoustic sensors are based on high precision microphones attached with tiny tips for capturing pressure pulsations at the local points [9]. Two of these sensors were used to conduct point-to-point measurements [9]. The perturbation mode that corresponds to the precessing vortex core was identified by analyzing the signal from the sensors located in diametrically opposite angular positions. Note that the possibility of using well-proven acoustic technology is another advantage of using air as the working medium.

(a)

Fig.1. A photo of the experimental setup (a), and a schematic of the test section (b)

Pressure fluctuations were recorded using a microphone Bruel\&Kjaer Type 2250, which was set half-way along the draft tube cone flush with the wall $(4.5 \mathrm{~cm}$ below the swirler), Fig. 2. The signals from the microphones were digitized by an analog-to-digital converter L-card E-440. The experiments were conducted at a fixed impeller rotation frequency $f=2432 \mathrm{rpm}$ and a flowrate, changeable in the range from $Q=65$ to $216 \mathrm{~m}^{3} / \mathrm{h}$ with a step of $0.4 \mathrm{~m}^{3} / \mathrm{h}$. At each point, the signal detected by the acoustic sensors was digitized for 5 seconds with a sampling rate of $2 \mathrm{kHz}$. 
The LDA measurements of the axial and tangential fluid velocity components were performed at a cross-section in the draft-tube cone located at $3 \mathrm{~mm}$ below the cowl of the swirler, Fig. 2.

Fig.2. Locations of the LDA measuring cross section and of the microphone mounted in the casing.

\section{The models and numerical details}

\subsection{Turbulence models considered}

Flows in hydroturbine systems are characterized by several features which pose a challenge to the RANS models. The flows in the guide vanes and the rotating impeller are dominated by the bounding wall - blades and housing, and a strong favourable pressure gradient which for the design operating conditions (no flow separation) can be handled reasonably well with a well-tuned wall-integration (WIN) model that (for the runner) accounts properly for the rotation effects. Accurate predictions of the blade-tip leakage, the follow-up vortical wakes and flow separation on blades at part loads, require more advanced, anisotropy-accounting RANS models. The phenomena in a draft tube are different. Here the flow is governed by a strong swirl and a bluff-body recirculation zone behind the impeller hub, which is usually reinforced by the negative pressure due to the swirl. The 90 degree bend encountered in elbow draft tubes, with a subsequent circular-to-rectangular change of the cross-section further modify the flow especially at part loads creating very complex vortical patterns with additional recirculation and secondary flows. On the other hand, the walls are smooth, and despite a strong elbow curvature, the wall-adjacent azimuthal fluid velocity and turbulence seem to follow reasonably well the common wall scaling so that computations seem manageable by the common wall-integration schemes or, according to some publications, even with using the standard wall-function approach. The apparent success of relatively standard wall treatment could in part be explained by strong radial pressure gradients and by inviscid interactions that diminish the wall effects as well as the role of the employed turbulence model on the dominant vortical structures and the consequent mean flow pattern.

The above brief overview of some major phenomena encountered in hydromachinery illustrate the importance of choosing the proper RANS model, which depends on which phenomena are in the focus of the computations. As stated above, our focus is on the draft tube and, specifically, on the strong and coherent swirling structures, primarily the precessing 
vortex cores and especially on the undesirable twin-ropes and the associated strong pressure pulsations at part loads. The linear eddy-viscosity (LEVM) turbulence models, widely spread in engineering calculations, are known to describe poorly most features listed above. Nevertheless, we tested some popular models, all validated against a well-resolved dynamic LES, aimed at scrutinizing the ability of various models to capture the above mentioned specific structures and pressure oscillation in the considered draft-tube model.

The computations here reported were performed with the ANSYS-FLUENT code (version 14.5) using the built-in two popular eddy-viscosity ( $k$ - $\omega$-SST and the realizable $k-\varepsilon$ ), a Re-stress model (Launder-Reece and Rodi, LRR, with the linear pressure strain and wallecho terms), and a DES (based on the $k-\omega$ SST Menter's model). In parallel, for reference, LES with the WALE subgrid-scale viscosity model was carried out on finer grids.

The high-Re-number realizable $k$ - $\varepsilon$ model and the basic RSM were solved using the standard wall functions. A test with the enhanced wall treatment and the non-equilibrium wall function available in the FLUENT code produced no significant differences in the results. For the $k-\omega$ SST we used the enhanced-wall-treatment $\omega$-equation model (EWT- $\omega$ ).

A specific issue in the computer simulation of hydraulic turbines is the treatment of the rotating impeller and the rotor-stator interaction. Several approaches can be found in the literature, i.e. dynamic, sliding and moving grid methods, and those based on a moving reference frame. The latter is the most common and the simplest way to model the runner rotation. It assumes that the runner is fixed and the equations are solved in a rotating reference frame. This formulation is often referred to as the "frozen rotor" approach. In this paper, the modelling of the runner rotation was performed in the rotated reference frame for the runner zone. The obtained results are then rotated with the runner rotation speed and as such imposed as the inflow field as the inlet into the draft tube. The earlier test calculations proved that this approach is credible for describing the integral flow characteristics including the dominant flow pulsations $[4,10,11]$. A comparison with the computationally more demanding method using sliding meshes showed that for this type flows with a focus on draft tube dynamics the results are almost the same.

The computations were performed using the finite-volume method on structured grids. The coupling of the velocity and pressure fields for incompressible flow was ensured using the SIMPLE-C procedure. For the URANS models the convective terms in all equation were discretized by a second-order upwind scheme, whereas the second-order central difference scheme was used for LES and DES (in the LES region). The time derivatives were approximated by an implicit second-order scheme. The time step was set from the condition CFL $<2$, but in the bulk of the draft tube the CFL number was less than 1.0. Some peaks exceeding the value of 2.0 appeared close to the guide-vane and impeller blades. The statistics was gathered for each run during about $20 \mathrm{sec}$ of real time.

\subsection{Computational domain, boundary conditions and grids}

The computational domain included all the elements constituting the turbine system (guide vane, impeller and draft tube) except for the intake channel (Fig.1). Since the intake channel was not considered in the calculations, the boundary conditions were set at the inlet to the turbine guide vanes by imposing a fixed flowrate measured in the experiment. As the LES 
method requires initial time-dependent forcing mimicking turbulence, random velocity fluctuations were imposed at the inlet, generated by the method proposed in [12].

The computations have been carried out using structured grids with the total number of 2, 6 and to $19.3 \mathrm{M}$ (million) nodes for the whole domain. The grid was clustered around the impeller blades, guide vanes and the draft tube walls (Fig. 3). The average distance of the wall-nearest grid nodes normalized by the wall units, $y_{1}^{+}$, for the $2 \mathrm{M}$ grid was 9 , for the $6 \mathrm{M}$ grid 1.25 and for the $19.3 \mathrm{M}$ grid it was 1.05 . The corresponding minimum and maximum values are given in Table 1, together with the proportion of the wall surface bounding the computational domain where $y_{1}^{+}$exceeds 2.0. As seen, for the coarse grid of $2 \mathrm{M}$ cells, this portion is $85 \%$, thus disqualifying this grid for the LES. For the $6 \mathrm{M}$ grid, the maximum value of $y^{+}$was 23 , and the minimum 0.02 with the wall-surface area for which $y_{1}^{+}$exceeds 2 being $23 \%$ of the entire surface. Likewise, for the $19.3 \mathrm{M}$ grid, the maximum $y_{1}^{+}$is 4.1 , the minimum 0.003 . And the wall-area proportion where $y^{+}$exceeds 2 , is only $8 \%$, confirming a sufficient near-wall resolution. An impression of the near-wall grid resolution is given in Fig. 4. Interestingly, the LES on a 6 mill grid produced the main flow features - the pressure pulsations, velocity profiles and the rms of their fluctuations in good agreement with results for 19.3 mill cells.
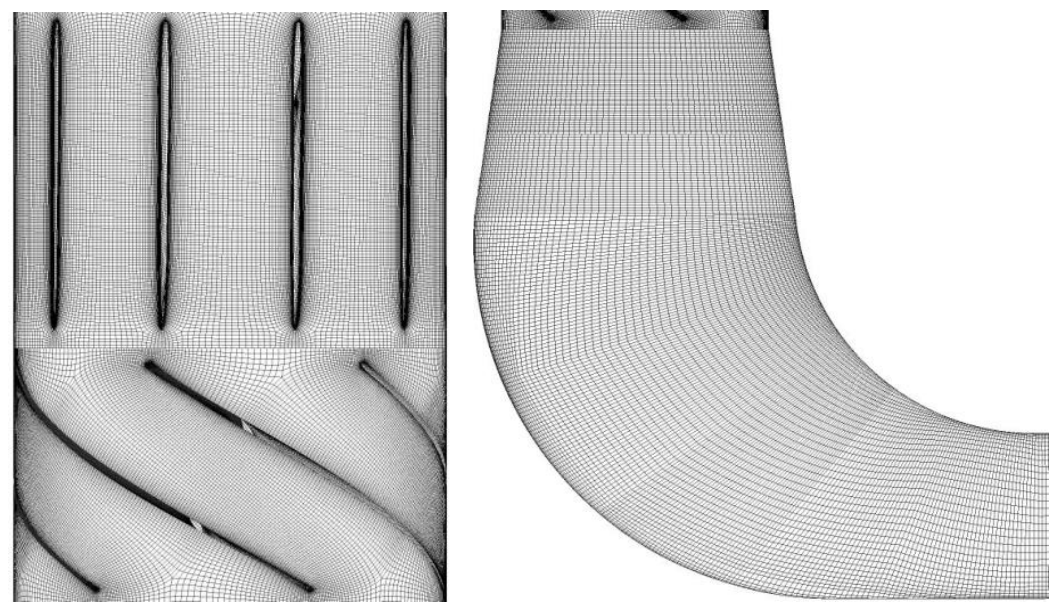

Fig.3. Views of the computational grid with 6 million nodes in the turbine model (guide vanes and runner) and its draft tube.
(a) $2 \mathrm{M}$ cells
(b) $6 \mathrm{M}$ cells
(c) $19.3 \mathrm{M}$ cells 

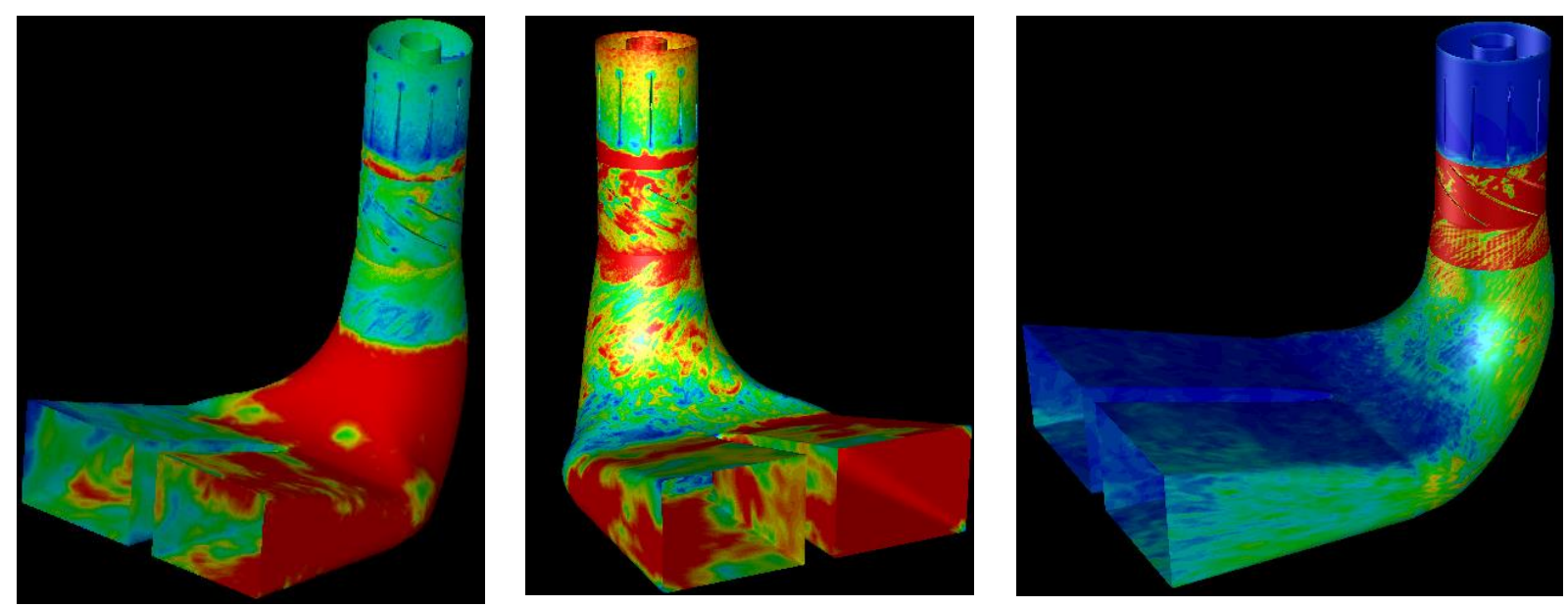

Fig. 4. Distribution of the wall-nearest grid node $y_{1}^{+}$on the walls for different meshes. Red colour indicates: (a) $y_{1}^{+}>20$, (b) $y_{1}^{+}>10$, (c) $y_{1}^{+}>1$.

To check further the grid resolution, the characteristic cell size $\Delta=(\Delta x \times \Delta y \times \Delta z)^{1 / 3}$ was compared with the Kolmogorov dissipative scale $L_{K}=\left(v^{3} / \varepsilon\right)^{1 / 4}$, the energy containing turbulence length scale $L_{t}=k^{3 / 2} / \varepsilon$, and the generalized Corrsin "shear" scale $L_{s}=\left(\varepsilon / S^{3}\right)^{1 / 2}$ (where $v$ is the kinematic viscosity, $k$ is the turbulent kinetic energy, $\varepsilon$ its dissipation rate (obtained from RSM), and $S=\left(S_{i j} S_{i j}\right)^{1 / 2}$ is the strain modulus. The shear scale, characterizing the scale of the turbulence energy production, was found by Picano and Hanjalic (2012) to serve well as a criterion for mesh resolution in free flows: when the grid-cell size was kept smaller than the shear scale (nominally $\Delta / L_{s}<1.0$, practically $\leq 0.8$, relevant primarily in high-shear regions), the LES of a free round jet reproduced well the mean flow and turbulence statistics at very high Re number using a very coarse grid despite the cell sizes being two order of magnitude larger than the Kolmogorov scale. Admittedly, no test is yet available for the near-wall high-shear region, but one can anticipate that a similar criterion would hold. Table 1 summarizes the main parameters for the three grids considered.

Table 1. Grid parameters for different models considered

\begin{tabular}{|c|c|c|c|c|c|c|c|}
\hline Grid/models & $y_{1, \min }^{+}$ & $y_{1, \max }^{+}$ & $y_{1, \text { aver }}^{+}$ & $\begin{array}{c}\text { Wall \% } \\
y_{1}^{+}>2\end{array}$ & $\Delta / L_{K}$ & $\Delta / L_{S}$ & $\Delta / L_{t}$ \\
\hline $2 \times 10^{6}($ RANS, DES) & 1.5 & 110 & 9.0 & $85 \%$ & & & \\
\hline $6 \times 10^{6}($ RANS, LES $)$ & 0.02 & 23 & 1.25 & $23 \%$ & $12-20$ & $0.1-3.0$ & $0.02-0.11$ \\
\hline $19.3 \times 10^{6}($ LES $)$ & 0.003 & 4.1 & 1.05 & $8 \%$ & $4.5-10$ & $0.04-1.1$ & $0.008-0.04$ \\
\hline
\end{tabular}

Figures 5 shows a comparison of different scale ratios for LES for the two fine grids in the flow-normal cross section of diffusor located at $3 \mathrm{~mm}$ below the impeller. As can be seen, 
for the grid $6 \mathrm{M}$ the ratio $\Delta / L_{K}$ of around 15 to 20 , whereas for the grid with $19.3 \mathrm{M}$ cells $\Delta / L_{K}$ is less everywhere than 10 , except for some sharp peaks very close to the walls in both cases. Interestingly, the cell-size to shear-scale ration $\Delta / L_{S}$ for both grids is below 1.0, except in the core region for the $6 \mathrm{M}$ grid, and of course very close to the wall for both meshes. This is in agreement with the findings in [15], which shows that the LES method can return satisfactory predictions when $\Delta / L_{S}$ is less than 1.0.
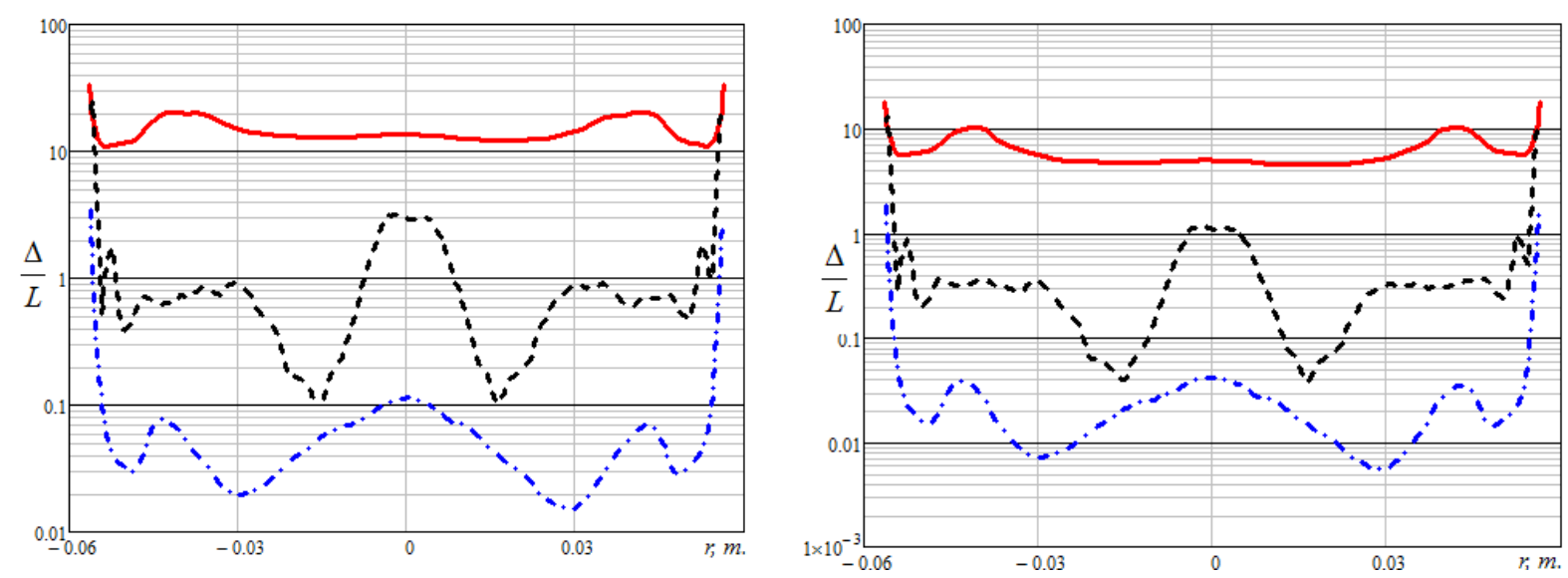

Fig. 5 Grid size compared with turbulence length scales in the middle plane of the horizontal draft-tube cross- section for $6 \mathrm{M}$ grid (left) and $19.3 \mathrm{M}$ grid (right). Top (red curve) $L=L_{K}$ (Kolmogorov scale), middle (black) $L=L_{s}$ (shear scale), bottom (blue) $L=L_{t}$ (energycontaining scale).

\subsection{Reference large eddy-simulations: experimental verification}

The here reported LES were aimed first to establish more comprehensive reference data beyond what is available from the experiment, but also to test the LES grid sensitivity, especially its performance on relatively coarse meshes that do not satisfy the common LES grid resolution criteria, with a view of possible application to real-scale hydromachinery. Surprisingly, the mean velocity profiles obtained with LES on very different grids, $6 \mathrm{M}$ and 19.3M shown in Fig. 6 (top) agree very well in between and also with the experiments. A difference appears in the spectra, but primarily at high wave numbers, which apparently does not affect mush the mean flow characteristics, Fig. 6 (bottom). The successful LES reproduction of the main flow features in the flow considered using a relatively coarse mesh of 6 million nodes can be explained by the fact that flow is dominated by the large coherent spiralling vortex (ropes), and thus not much affected by the unresolved the small-scale motion. Another explanation can be traced in grid optimization made to satisfy the shear-scale criterion, $\Delta / L_{S}<1$, congruent with the similar finding for LES of a free round jet reported in [15]. 

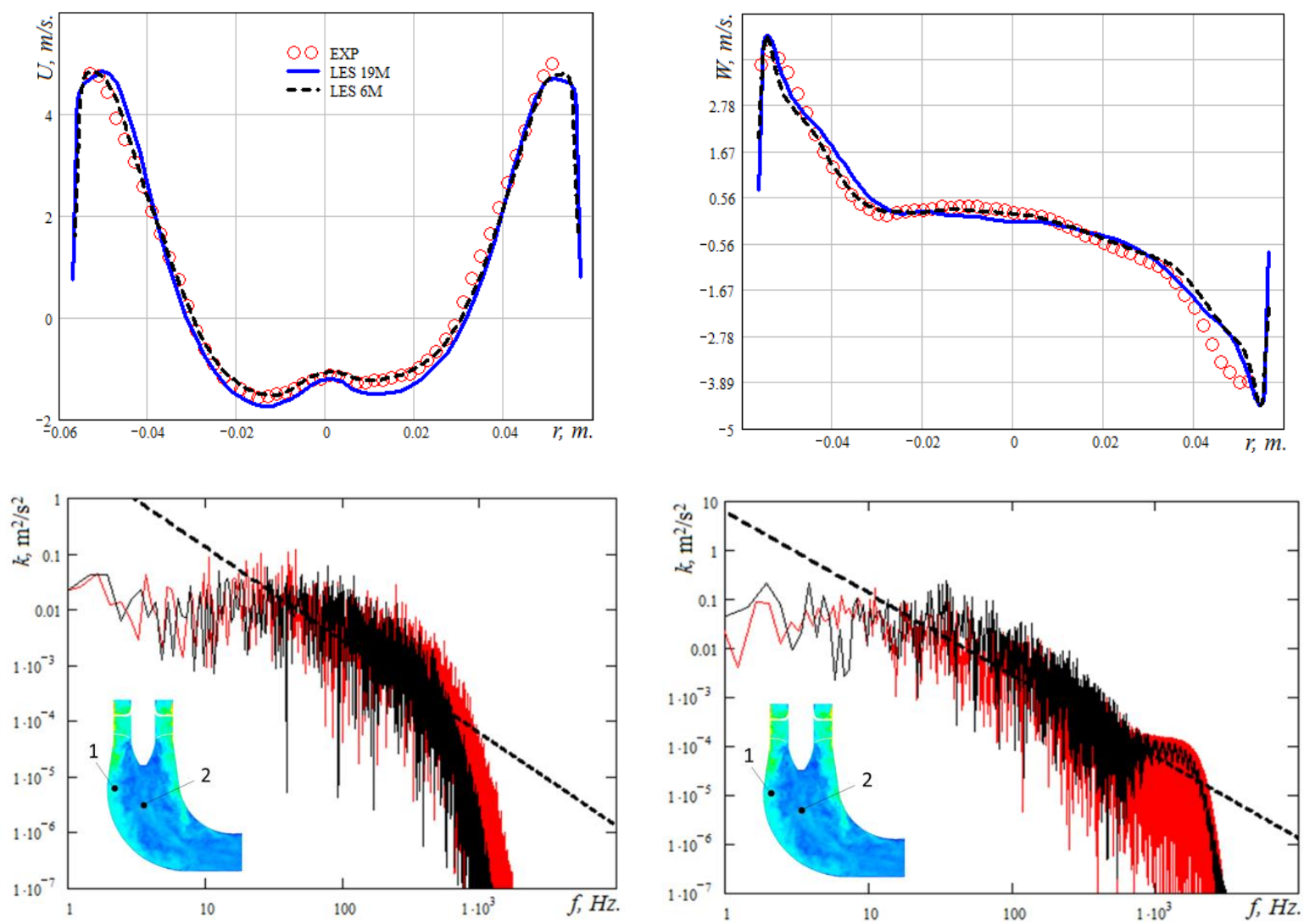

Fig. 6 Comparison of LES on $6 \mathrm{M}$ and $19.3 \mathrm{M}$ grids for the low part load operation, $Q / Q_{n}=39 \%$. Top: Axial (left) and tangential (right) mean velocity profiles. Bottom: spectra at location 1(left) and 2 (right); black (dark) $6 \mathrm{M}$ grid, red (gray) $19.3 \mathrm{M}$. grid.

A further support for the above is given in Fig. 7, which compares the axial and tangential velocity components obtained from LES on $6 \mathrm{M}$ grid, with the measurements at the reference cross-section for three different loads, the nominal load $Q_{n}=50 \mathrm{l} / \mathrm{s}$ and two off-design conditions, $Q=40$ and 55 1/s. For all three cases, agreement appears quite satisfactory save for some unexplainable difference for the nominal regime (middle figures).

In what follows we shall, therefore use the LES on $6 \mathrm{M}$ grid for comparison and validation the RANS models obtained on $2 \mathrm{M}$ and $6 \mathrm{M}$ grids for the low part load of $39 \%$. 

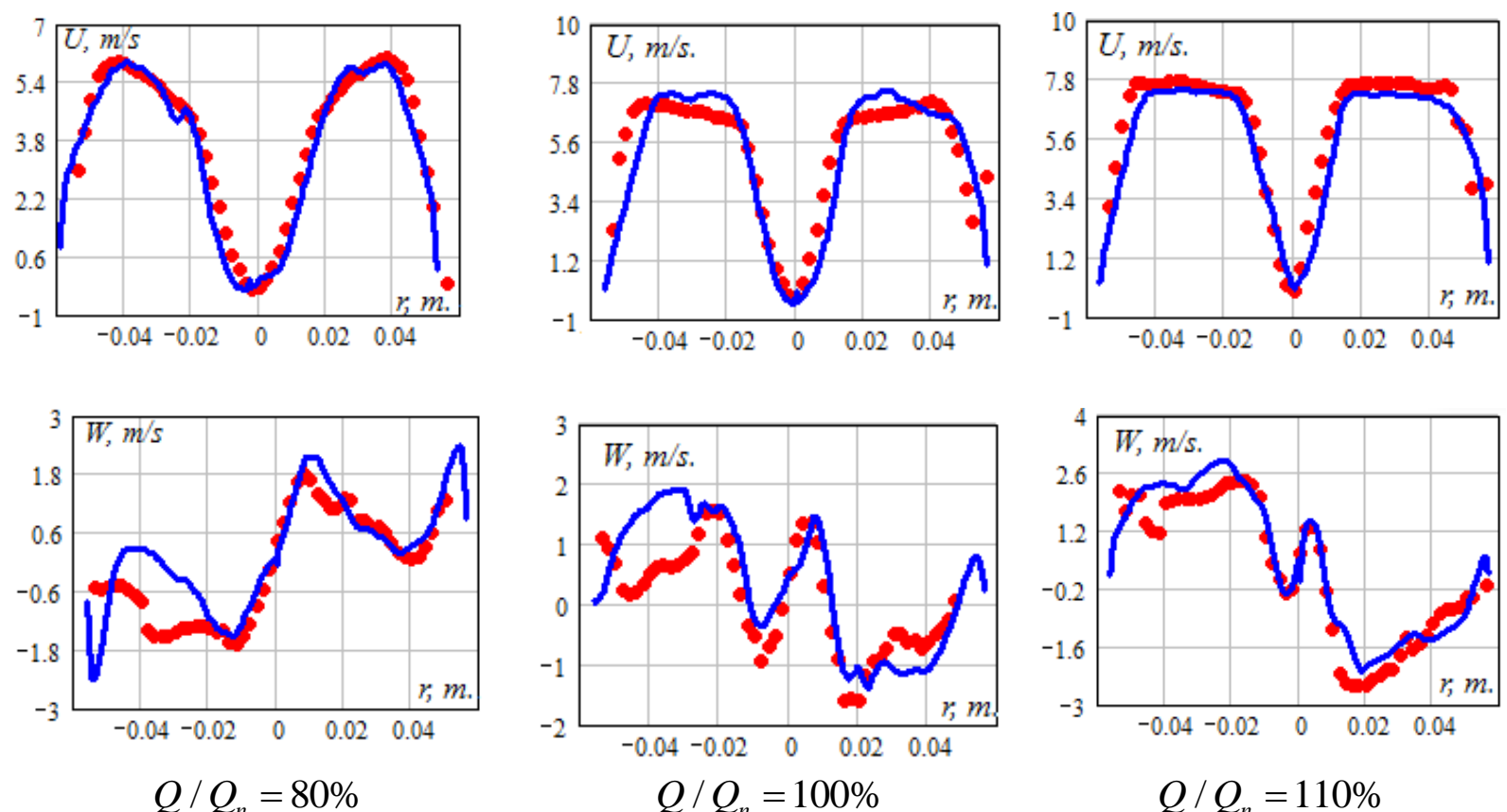

Fig. 7. LES on $6 \mathrm{M}$ grid (solid lines) compared with experiments (symbols) in the draft tube diffusor at $3 \mathrm{~mm}$ below the runner cone for three operating regimes close to the nominal (BEP). Top: axial mean velocity, bottom: tangential mean velocity.

\section{A scrutiny of the URANS performance for a part load}

We present now first a summary of performance of various URANS model on a typical RANS grid of $2 \mathrm{M}$ nodes and compare with the LES on $6 \mathrm{M}$ and DES on $2 \mathrm{M}$ grid for the low part load of $Q / Q_{n} \approx 0.4$. The time-averaged streamline patterns and colour contours of the fields of the axial velocity in Fig. 8 clearly show that the linear eddy viscosity models (LEVM) incorrectly describe this flow, whereas the RSM, DES and LES all give very similar results. This is further substantiated by a quantitative comparison with experiments of the profiles of the axial and tangential velocity components and the rms of their fluctuations at the $3 \mathrm{~mm}$ cross-section, Figs 9 and 10. It is noted that the LES reproduced best the experimental data, but the DES and RSM (on 2M grid) show also good agreement. In contrast, both EVMs notably failed, as shown by typical (unphysical) solid-body profiles of tangential velocity and a wrong recirculation pattern. Moreover, both LEVMs, the $k-\varepsilon$ realizable and $k-\omega$ SST models on the $2 \mathrm{M}$ grid could not maintain the unsteadiness and resulted in stationary solutions failing to capture the twin rope precessing structures as discussed below and shown in Fig. 16. 
(a) $\mathrm{k}-\varepsilon$ realis. $(2 \mathrm{M})$

(b) $\operatorname{RSM}(2 \mathrm{M})$

(c) DES (2M)

(d) LES (6M)
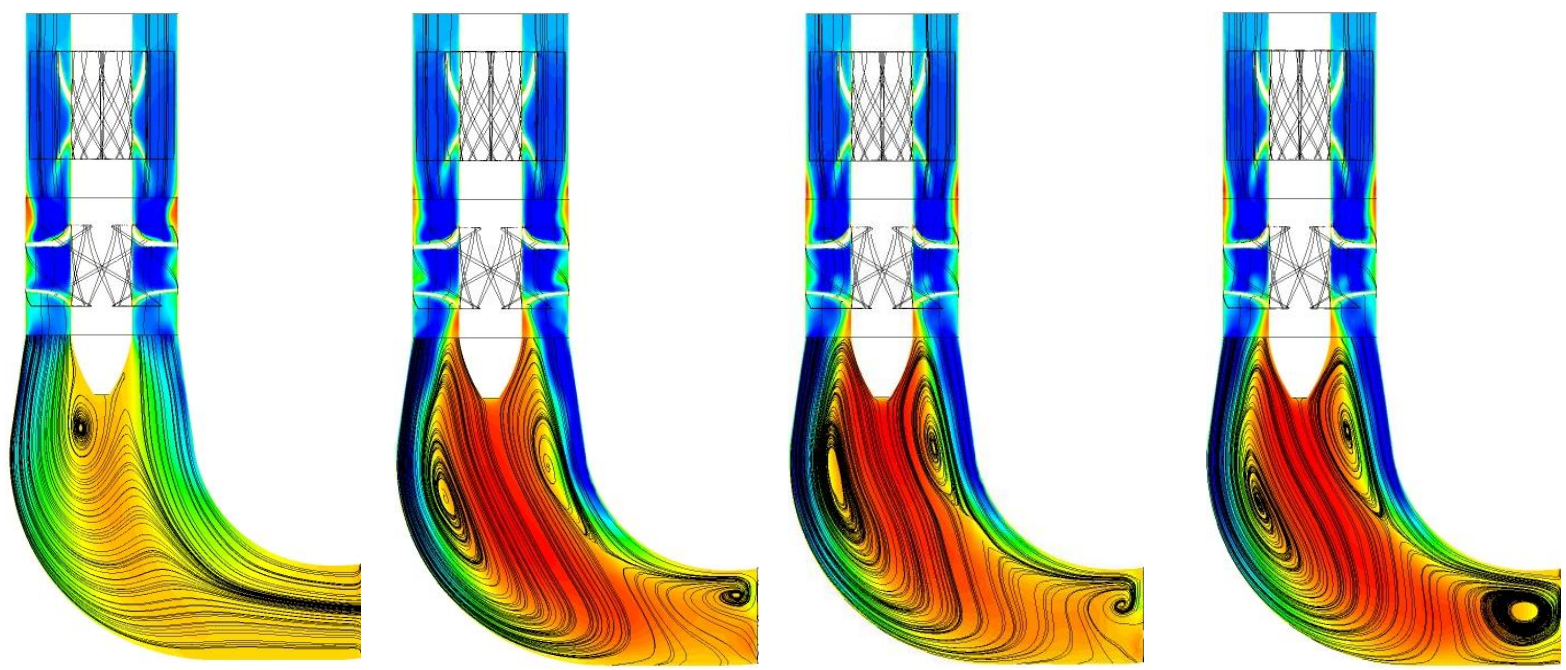

Fig. 8. Time-averaged axial velocity field and streamlines. The colour denotes the velocity magnitude.
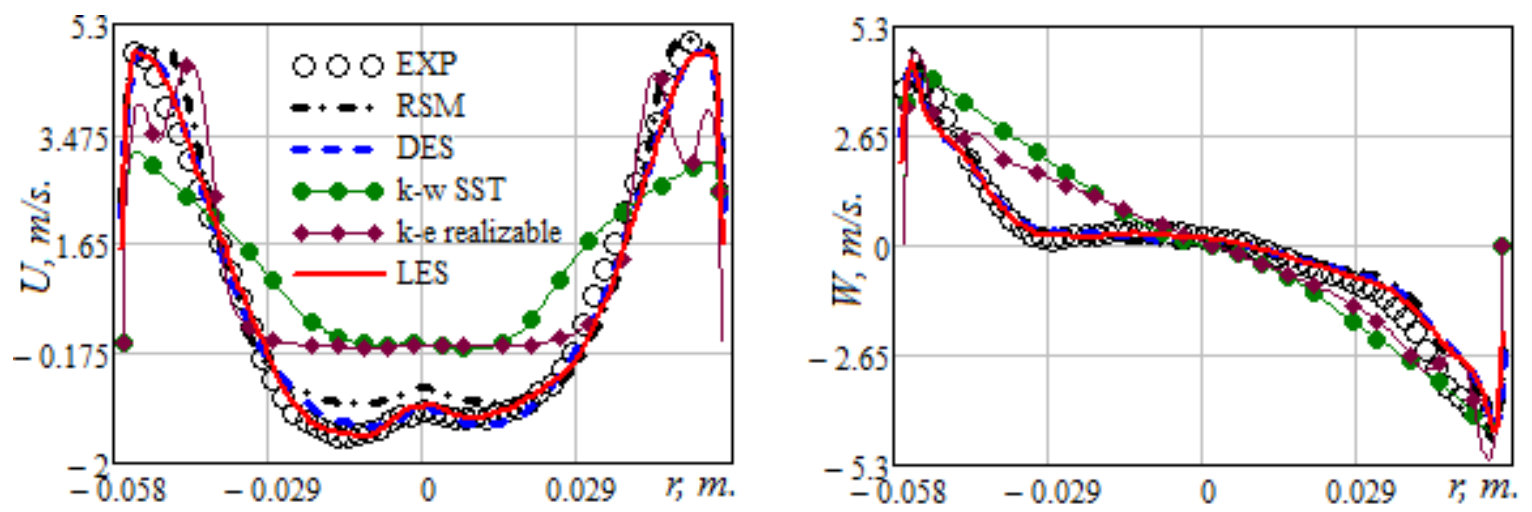

Fig. 9. Axial (a) and tangential (b) velocity component in the draft tube diffusor at $3 \mathrm{~mm}$ behind the cone, obtained by different turbulence models. Grids: (U)RANS and DES 2M, LES 6M. 

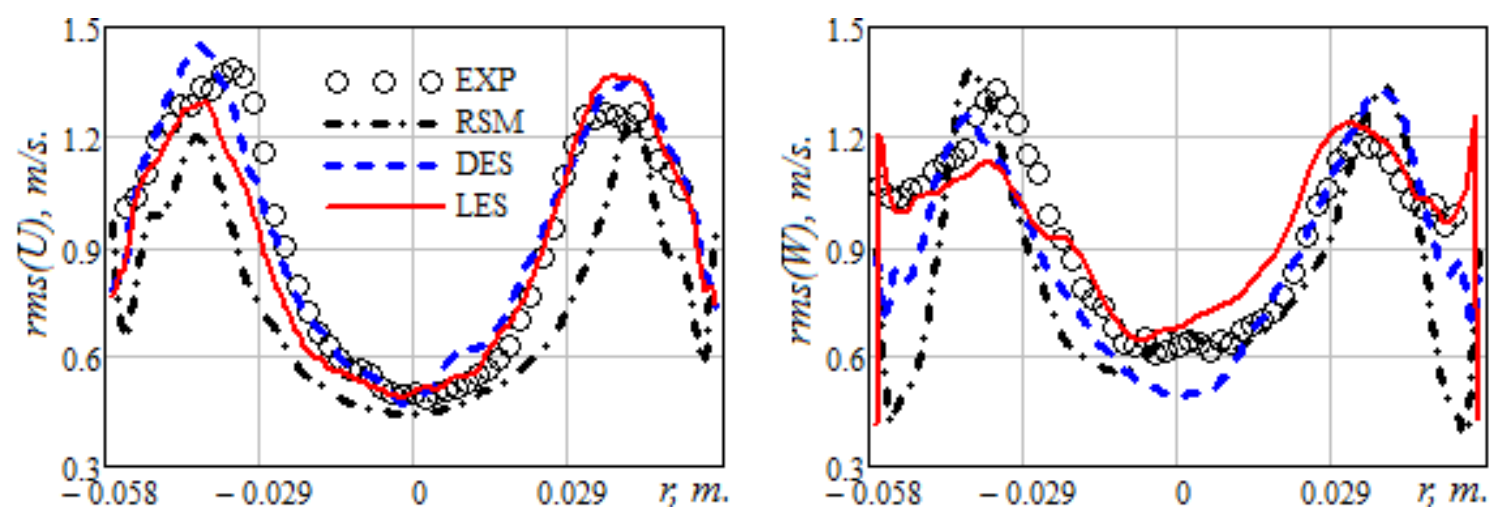

Fig. 10. The root-mean-square ( $r m s$ ) of the axial (a) and tangential (b) velocity fluctuations in the draft tube diffusor at $3 \mathrm{~mm}$ behind the cone obtained by different turbulence models. Grids: (U)RANS and DES 2M, LES 6M.

The same prediction quality and trend are observed in the cross section located at $53 \mathrm{~mm}$ below the impeller as shown by the velocity profiles and their fluctuations in Figs 11 and 12 . Due to the lack of experimental data at this cross-section we cannot verify the computational data. However, it can be seen that just as in Figs 9 and 10 for the cross-section of $3 \mathrm{~mm}$, the RSM and DES models are in good agreement with the reference LES results, whereas the results obtained by the two linear EVM models differ significantly showing the same shortcomings as in Figs 9 and 10.
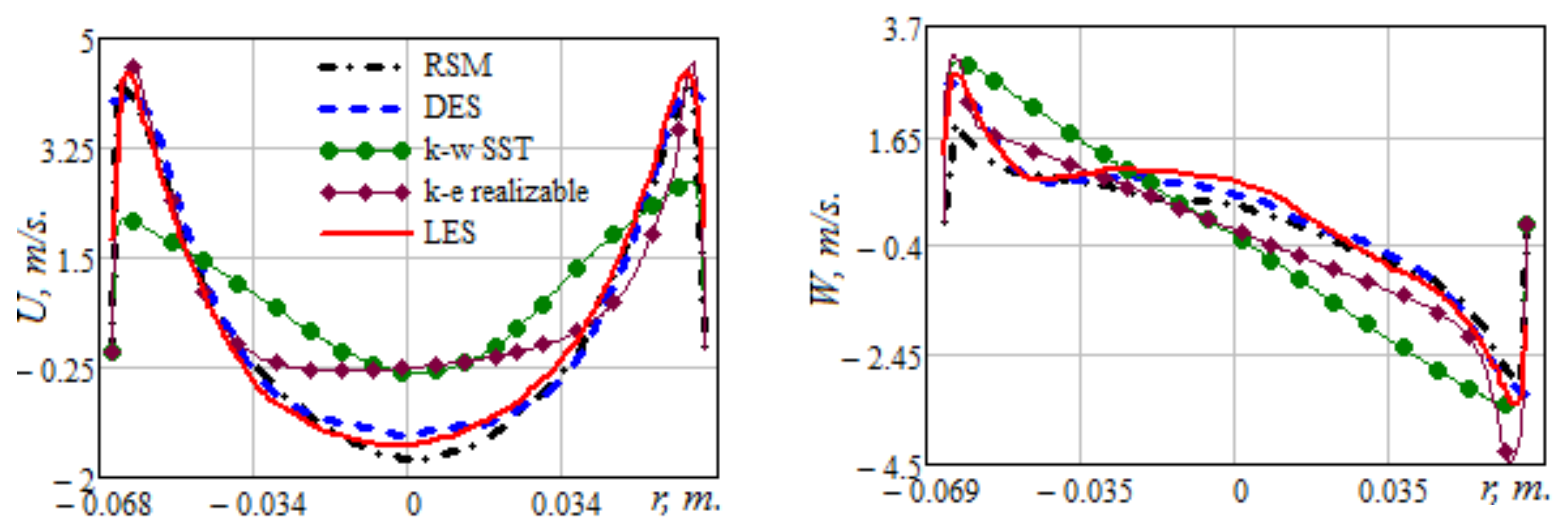

Fig. 11. Profile of the axial (a) and tangential (b) velocity component in the draft tube diffusor at $53 \mathrm{~mm}$ behind the cone obtained by different turbulence models: (U)RANS and DES 2M, LES 6M. 

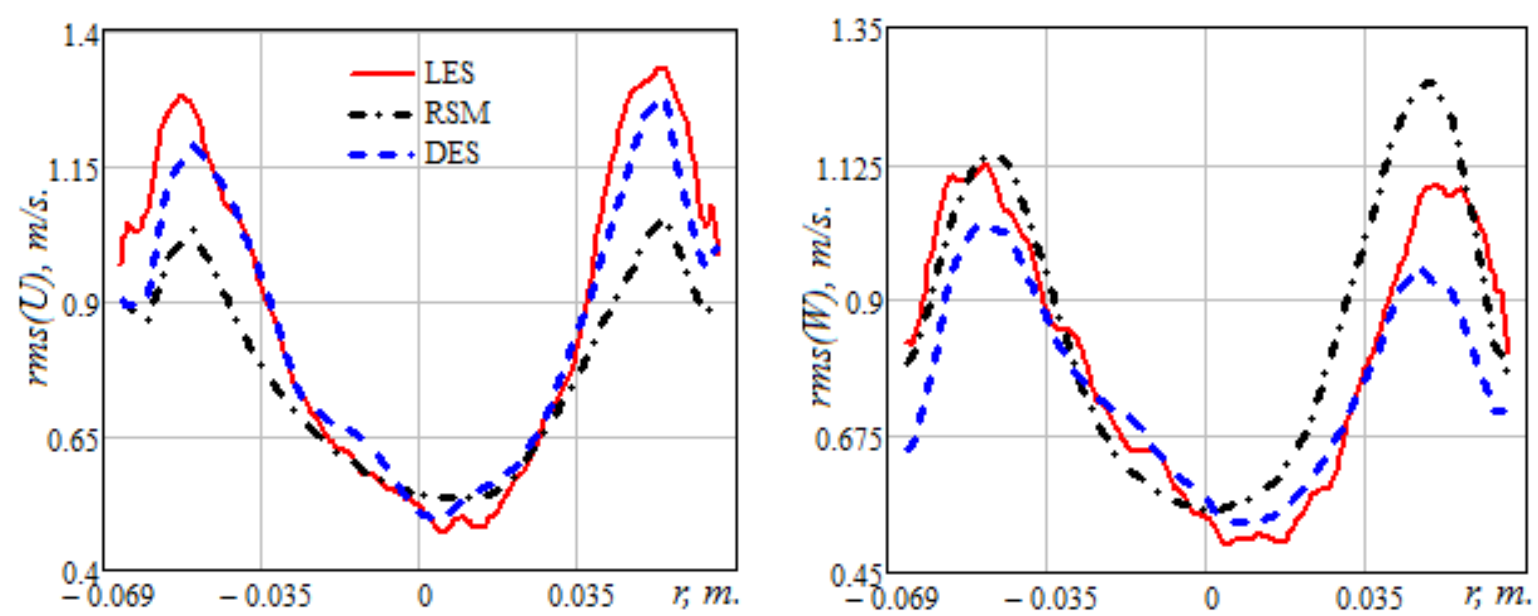

Fig. 12. The root-mean-square ( $r m s$ ) of the axial (a) and tangential (b) velocity fluctuations in the draft tube at $53 \mathrm{~mm}$ behind the cone obtained by different turbulence models. (U)RANS and DES 2M, LES 6M.

However, using a finer grid of $6 \mathrm{M}$ notably improved the performances of the two LEVMs. This time the URANS computations maintained the unsteadiness, capture the characteristic time-averaged streamline pattern and the associated mean velocity profiles, as illustrated in Fig. 13 (here shown for the $k$ - $\omega$ SST model), comparable to RSM and DES on $2 \mathrm{M}$ in Fig. 8. As seen in Fig. 13, the URANS computations on $2 \mathrm{M}$ grid resulted in a steady solution which differs from the LES solutions (but also from the k- $\varepsilon$ realizable model, Fig. 9), whereas the same model on the $6 \mathrm{M}$ grid produced unsteady solutions, which after averaging, come much closer to the reference LES pattern.

(a) $k-\omega \operatorname{SST}(2 \mathrm{M})$

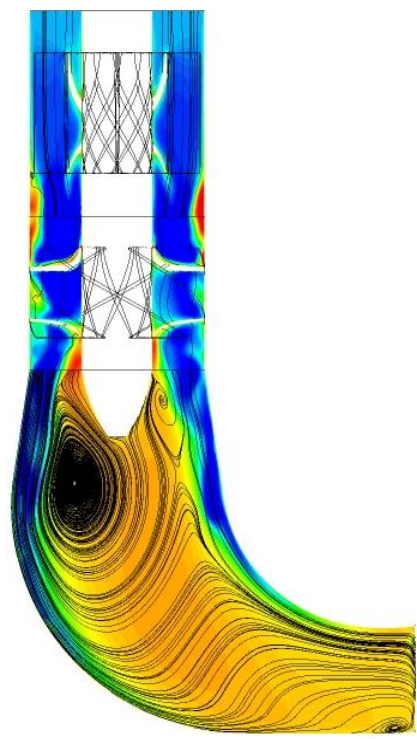

(b) URANS $k-\omega \mathrm{SST}(6 \mathrm{M})$

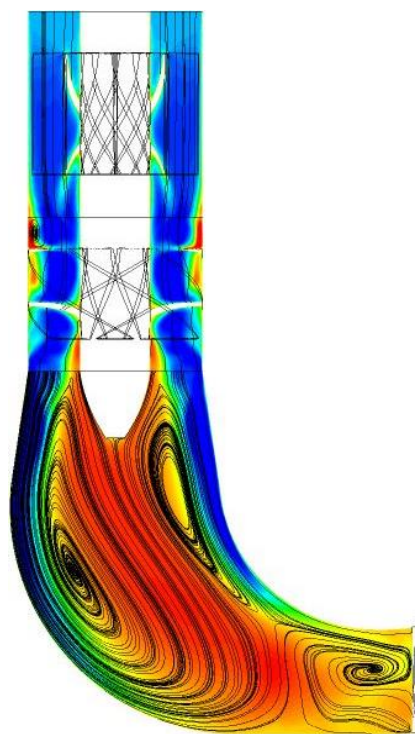

(c) LES (6M)

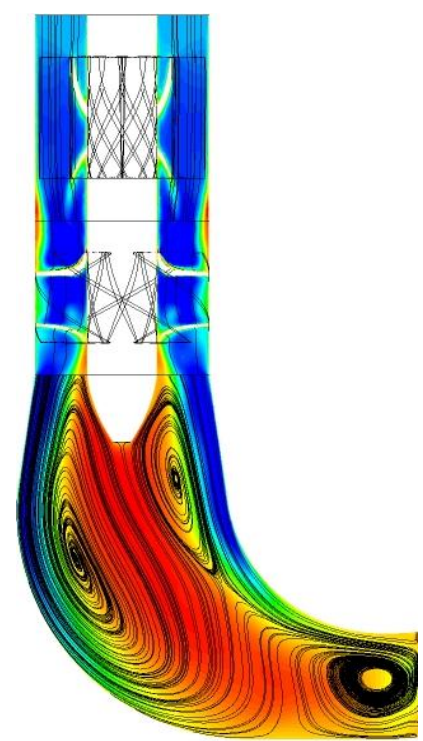

Fig. 13. Time-averaged axial velocity field and streamlines from (U)RANS EVMs compared with LES. 
Figure 14 confirms the above by comparison of the mean velocity and turbulent kinetic energy with the measurements. A striking consequence of the failure of LEVMs on the coarser grid is seen in the profiles of the kinetic energy (Fig. 14 bottom left), where the failure to retain the unsteadiness and to resolve the fluctuating velocities led to a compensation by an erroneous modeled stress field.
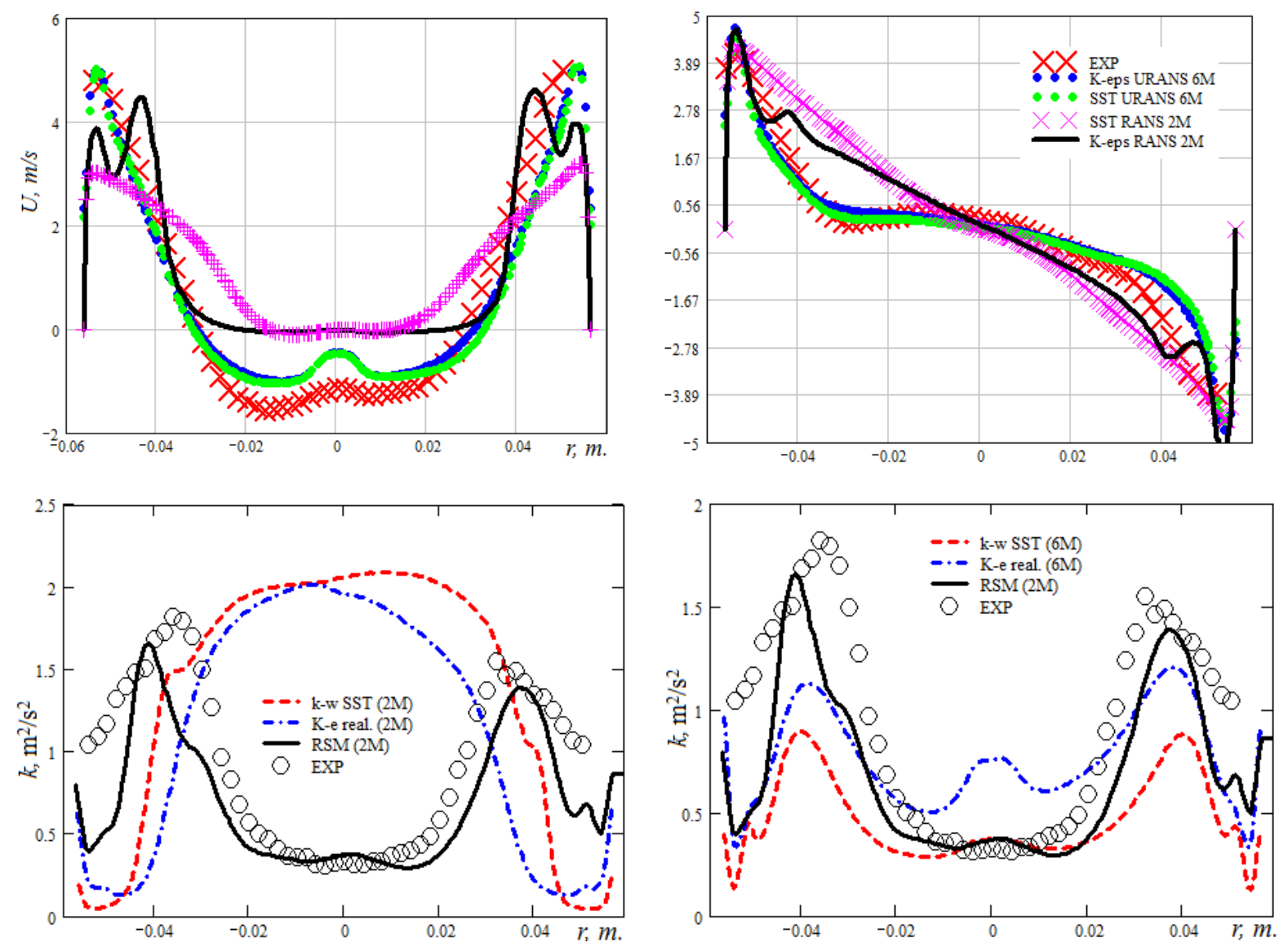

Fig. 14. Comparison of (U)RANS in draft tube at $3 \mathrm{~mm}$ cross-section on $2 \mathrm{M}$ and $6 \mathrm{M}$ grids. Top: axial (left) and tangential (right) velocity component. Bottom: total (resolved plus modelled) turbulent kinetic energy; left: $2 \mathrm{M}$ grid; right: EVMs on 6M and RSM on $2 \mathrm{M}$ grid.

\section{Vortical ropes and pressure pulsations}

We return now to discuss the potential and limitation of the considered RANS models in capturing the precessing vortex structures and the associated pressure pulsations in the draft tube, which is of the prime concern for design and operation of hydroturbine systems. But before discussing the phenomenon associated with highly unsteady regimes that are far from the best operating point, usually at strongly reduced flowrates or overloads, we show first the computer visualization of the typical vortex structures below the turbine runner at some loads that are close to the nominal, best-efficiency regime. Figure 15 displays the vortex ropes identified by the pressure isosurface for flowrates of 40, 50 and $551 / \mathrm{s}$, corresponding 
respectively to $80 \%, 100 \%$ and $110 \%$ of the nominal load. Note that for the same three regimes the comparison of the experimental and LES (on 6M mesh) results for the axial and tangential mean velocity component shown in Fig. 7 display generally good agreement.

The vortex rope patterns for the nominal regime and at a mild overload ( $Q_{n}$ and $1.1 Q_{n}$ ) look similar, characterised by a central non-precessing vortex, surprisingly visibly amplified and elongated for $Q / Q_{n}=1.1$. In both cases, a mild precessing vortex core is generated at the tail of the central vortex. However at a part load $Q / Q_{n}=0.8$, the orderly central vortex has lost its identity being replaced by multiple swirling structures populated by a range of smallerscale vortices, which will presumably form distinct precessing single or twin vortex ropes as the flowrate is further diminished.
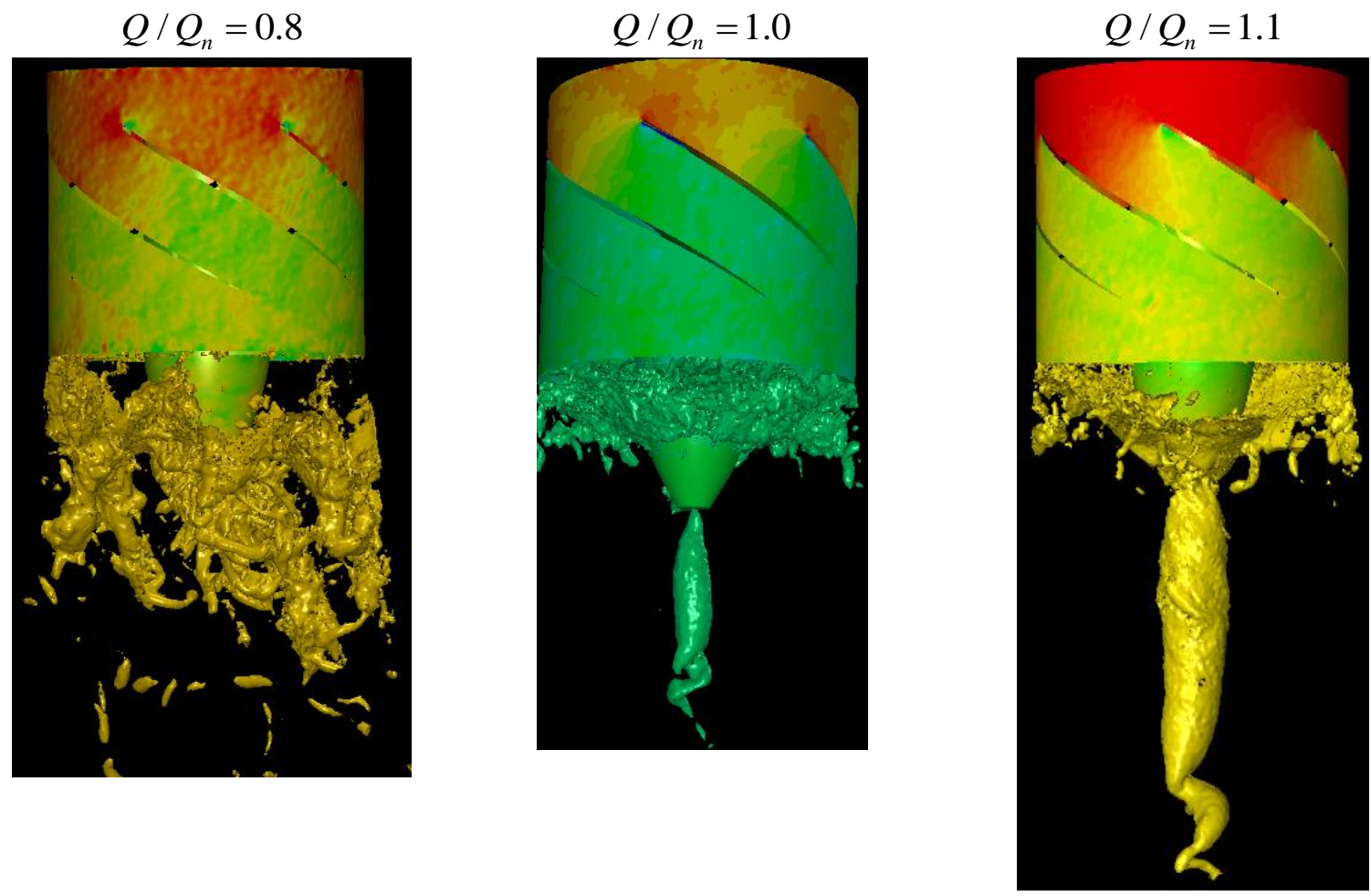

Fig. 15. The instantaneous vortex ropes identified by the pressure iso-surfaces for three operating regimes at and relatively close to the nominal.

We move now to discuss the part load of $39 \%$ of the nominal. It is first recalled that precession of the vortex core occurs under partial load (in the turbine model here considered at a discharge rate of $0.2-0.85$ of the optimal value), where the flow has a considerable residual swirl. Vortex breakdown in such a flow leads to the formation of the recirculation zone at the flow axis and the rotation of the corkscrew vortex rope (often visible by local evaporation or/and dissolved air) around it. Figure 16 shows selected snapshots of the typical vortex ropes and their structures after the impeller, captured by different models and compared with experimental visualization. The ropes are, as before, identified by the instantaneous isosurfaces of a selected value of pressure. 
(a) Experiment

Skripkin et al (2015) (b) LES

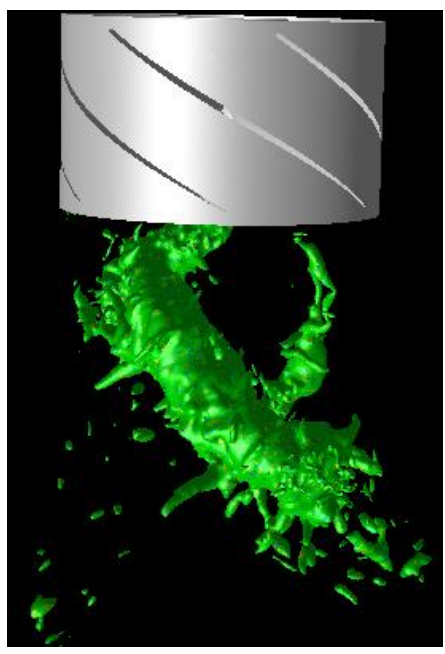

(e) URANS SST (6M)

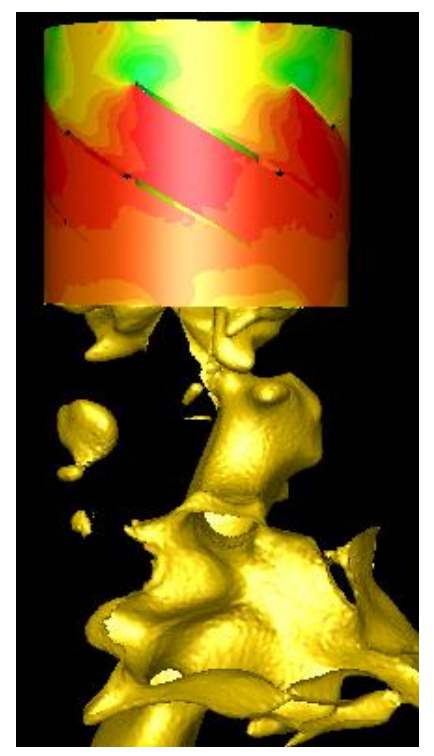

(c) RSM

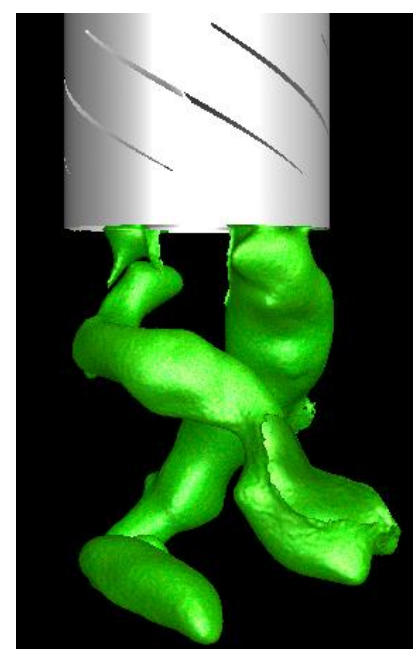

(f) $\operatorname{LES} \mathrm{Q}=1 \times 10^{6}$

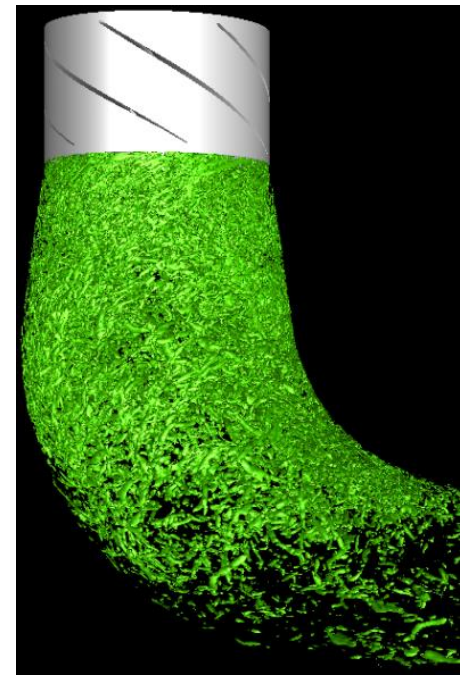

Fig. 16. Snapshots of the instantaneous vortex ropes identified by the pressure isosurfaces, obtained by different models. Bottom right: An overall impression of the fine vortical structures visualised by a high value of the vortex eduction criterion $\mathrm{Q}$.

As seen in Fig. 16a and 16b, the experiments and LES show that at the part load regime here considered the vortex structures take the form of a twin rope. Their formation influences the whole flow field, creating a strong back-flow in the central region of the draft tube as already seen in Figs 8 and 13, as well as in the velocity profiles in Figs. 9, 11 and 14 [10, 1316]. Moreover, Figs 8 and 13 show a separate spanwise oriented detached vortex at the end of the draft-tube diffusor which most probably is not stationary, but most likely oscillates in the streamwise direction. A major consequence of the formation of helical ropes and associated unsteady structures are the pressure pulsation in the draft tube which propagate throughout the whole turbine and its system. The precessing vortex rope itself generates intense flow- 
induced pulsations, which pose a serious threat to the reliability and safety of operation of hydraulic turbine equipment. Pressure pulsations lead to vibrations of the turbine structure that can lead to damage, especially in the case of resonance. Pressure pulsations, generated by vortex ropes may also affect cavitation processes.

It is interesting that the Re-stress LRR model captures well the phenomenon, albeit with smoothed fine-scale structures, Fig. 16c. However, despite maintaining the unsteadiness and reproducing reasonably well the man velocity components and their rms (Figs 13 and 14), none of the tested LEVM URANS even on the $6 \mathrm{M}$ grid, reproduced the twin-rope vortical pattern, as revealed in Figs $16 \mathrm{~d}$ and $16 \mathrm{e}$ ! The $k-\varepsilon$ realizable model gives one thick vortex showing little if any precessing, Fig.16d, and the SST model gives only a hint at possible double vortex initiation, but with only one, irregularly shaped structure with no indication of a spiralling helical pattern. Likewise, although the unsteady LEVM solutions capture some transient pressure pulsation, the intensity and the character are not in accord with the experiments or LES.

For practice, whether design or operation, it is vital to know the characteristic parameters of the pressure pulsations, primarily their intensity (especially the maximum amplitudes) and frequency. Since for the evaluation of these parameters the industry relies now more and more on the CFD computations, using most often licenced CFD codes, it is essential to know a priori which of the model and method can be relied upon to deliver these information. To this purpose, in Fig. 17 we compare the calculated and measured pressure fluctuations. The experimental pressure recordings are available from the measurements at the discrete points in the middle of the draft tube cone and flush with the wall $(4.5 \mathrm{~cm}$ below the swirled, Fig. 2).

Figure 17 (left) shows that the LES on 6M grid, and DES (not shown) and the RSM on 2 $\mathrm{M}$ grid return well the character of pulsations and the measured maximum peak-to-peak amplitude of 9.57 Pa: the LES signal is almost indistinguishable from the experiment with the peak-to-peak value of $10.1 \mathrm{~Pa}$, whereas the RSM gives a somewhat smoother recording, but with the right frequency and the maximum amplitude of 9.1 Pa. The latter finding is in a sense surprising, because it was previously considered by some researchers that the RSM usually returns lower amplitudes. In contrast, the LEVMs, despite maintaining the unsteadiness significantly underestimate the pressure pulsations, e.g. the URANS SST model gives a maximum peak-to-peak amplitude of $4.2 \mathrm{~Pa}$.
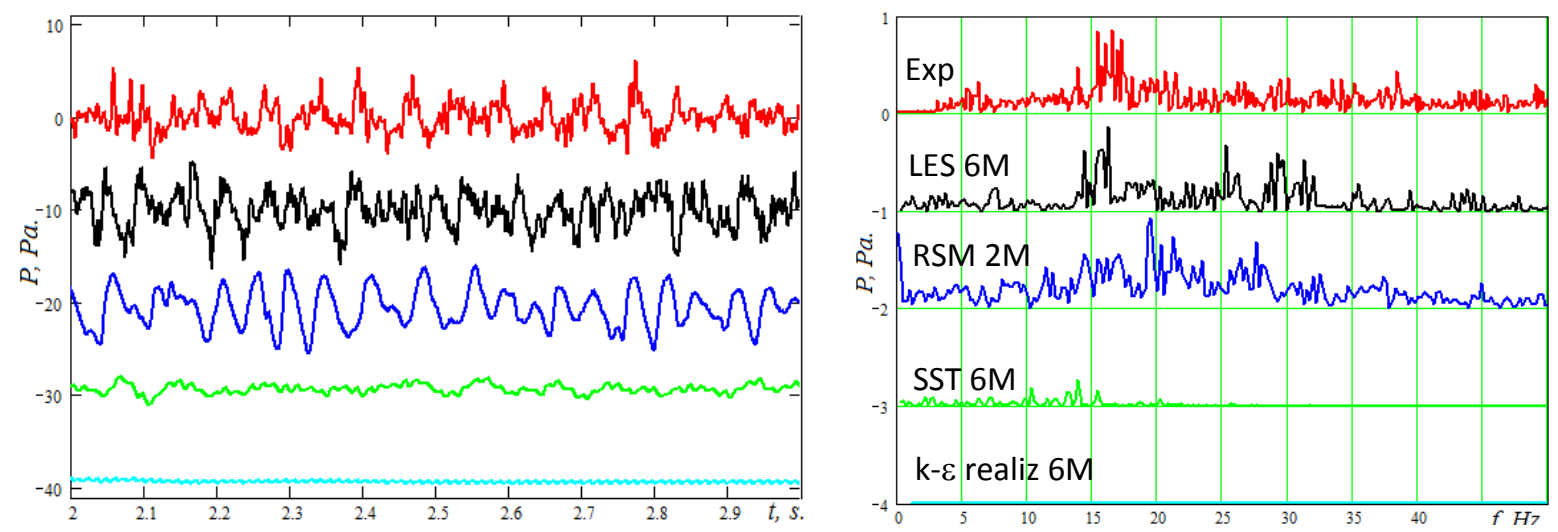

Fig. 17. Time-records (left) and spectra (right) of pressure pulsation at the sensor 
(microphone) location (Fig. 2). From top to bottom: experiment (red), LES 6M (black), RSM $2 \mathrm{M}$ (blue), URANS SST 6M (green) and URANS k- $\varepsilon$ realiz. 6M (emerald). The vertical-axis notations correspond to the experiments; for other curves it is shifted down in steps of 10 and $1.0 \mathrm{~Pa}$ respectively.

Thus, one of the main findings of the present research is the evidence that the second moment closure (Reynolds-stress model, RSM) even at the basic level (e.g. linear model of the pressure-strain correlation) and on a relative coarse gird (2 mill cells) is sufficiently receptive and sensitive to strong flow unsteadiness associated with vortex ropes and precessing vortex cores and can be used to estimate the level of the pressure pulsations in hydroturbine draft tubes.

Next to the amplitudes, it is also important to know the frequency of pressure pulsation, especially to identify the values associated with the dominant vortex structure, to avoid the potential resonance with eigen frequencies of the turbine and its equipment. A comparison of the measured and calculated frequency spectra is shown in Fig. 17 (right). As noted earlier, the twin rope structure in the draft tube immediately behind the impeller consists basically of two precessing helical vortices, which apparently have somewhat different frequencies. This is seen in the measured spectra (Fig. 17 right) which indeed has identifiable peaks at frequencies of 13.88 and $16.5 \mathrm{~Hz}$ corresponding to a more and less intense helix respectively. The LES seems to capture well the double-frequency character of the spectra with peaks at 14,36 and $16,22 \mathrm{~Hz}$ respectively, close to the experimental values, though indicating also some stronger activities at about 25 and $30 \mathrm{~Hz}$, which are not visible in the experimental recording. The RSM spectra look similar to LES with several peaks, the basic ones believed to correspond to the twin vortex rope being at frequencies of 14.38 and 19,2 Hz. Interestingly, for the locations considered (corresponding to those of wall pressure sensor points) neither the experiment nor any of the computations detected visibly the frequency corresponding to the impeller/blades rotation speed of $40.5 \mathrm{~Hz}$.

\section{Conclusions}

Two linear-eddy viscosity (the $k-\varepsilon$ realizable and the $k-\omega$ SST), the basic Reynolds-stress (LLR) RANS models, and the SST DES (all as available in the ANSYS-FLUENT CFD code), have been scrutinized against the well-resolved LES (on meshes with 6 and 19.3 M (million) nodes) and experiments in reproducing the unsteady flow, turbulence and vortical structures in an elbow-type draft tube of a 60:1 scaled down model Kaplan hydroturbine operating at a part load of $40 \%$ of the nominal flowrate. To generate the proper inflow conditions, the simulations encompass the guide-vanes, turbine impeller and the diffusor. The aim was to test the suitability of some popular (U)RANS model for coarse-grid industrial-purpose predictions of unsteady flows in hydroturbine systems operating in off-design regimes. The focus was on the dynamics of precessing helical vortex ropes and the associated pressure pulsations.

The computations showed that the two linear eddy viscosity models on $2 \mathrm{M}$ grid could not maintain the unsteadiness and failed to reproduce the essential flow features, but on a finer grid of $6 \mathrm{M}$ resulted in unsteady solutions with improved mean-flow patterns, though still 
failing to capture the twin-helix structures and the pressure pulsations. In contrast, the secondmoment closure (Re-stress) model on the same $2 \mathrm{M}$ mesh recovers well both the mean velocity field, the rms of its fluctuations, as well as the specific helical double rope structure in accord with experiments and LES. Similar performance is returned by DES.

It was also shown that the LES on a $6 \mathrm{M}$ and $19.3 \mathrm{M}$ grids performed similarly. This, to some extent surprising finding, is attributed to the fact that the cell size was optimized to be smaller than the Corrsin shear scale, while resolving the fine scales proved not to be essential for the flow dominated by strong large-scale coherent vortical structures.

\section{Acknowledgements.}

This work was supported by the Russian Science Foundation (grant №14-29-00203).

\section{References}

1. Doerfler P. System dynamics of the Francis turbine half load surge. 1982, Proc. IAHR Symp. on Hydraulic Machinery and Systems, Amsterdam, Netherlands.

2. Alligne S., Maruzewski P., Dinh T., Wang B., Fedorov A., Iosfin J. and Avellan F., 2010, Prediction of a Francis turbine prototype full load instability from investigations on the reduced scale model. Proc. IAHR Symp. on Hydraulic Machinery and Systems, Timisoara, Romania.

3. Cervantes M.J., Engstrom T.F., Gustavsson L.H., 2005, Proc. The third IAHR/ ERCOFTAC workshop on draft tube flows Turbine 99. Sweden, Porjus,. 193 p.

4. Minakov, A.V. Sentyabov, D.V. Platonov, A.A. Dekterev, A.A. Gavrilov, 2014, Numerical modeling of flow in the Francis-99 turbine with Reynolds stress model and detached eddy simulation method. Journal of Physics: Conference Series Volume 579, doi:10.1088/1742-6596/579/1/012004.

5. Bosioc Alin, Tanasa Constantin, Susan-Resiga Romeo, Muntean Sebastian. 2D LDV Measurements of Swirling Flow in a Simplified Draft Tube, Conf. Model. Fluid Flow. 2009.

6. Chen Chang-Kun, Nicolet Christophe, Yonezawa Koichi, Farhat Mohamed, Avellan Francois, Miyazawa Kazuyoshi, Tsujimoto Yoshinobu. Experimental Study and Numerical Simulation of Cavity Oscillation in a Diffuser with Swirling Flow, Int. J. Fluid Mach. Syst. Korean Fluid Machinery Association, - 2010. - Vol. 3, № 1. - P. 80-90.

7. Susan-Resiga Romeo, Muntean Sebastian, Tanasa Constantin, Bosioc Alin. Hydrodynamic Design and Analysis of a Swirling Flow Generator // Proc. 4th Ger. Rom. Work. Turbomach. Hydrodyn. (GRoWTH), June 12-15, 2008, Stuttgart, Ger.

8. Kuibin P.A., Litvinov I.V., Sonin V.I., Ustimenko A.S., Shtork S.I. Modelling inlet flow in draft tube for different regimes of hydro turbine operation // Vestnik Novosibirsk State University. Series: Physics., 2016, in press

9. Litvinov I. V., Shtork S.I., Kuibin P.A., Alekseenko S. V., Hanjalic K., 2013, Experimental study and analytical reconstruction of precessing vortex in a tangential swirler, Int. J. Heat Fluid Flow 42: 251-264.

10. Minakov, A.V., Platonov, D.V., Dekterev, A.A., Sentyabov, A.V., Zakharov, A.V., 2015,. The numerical simulation of low frequency pressure pulsations in the high-head Francis turbine. Computers \& Fluids. 111: 197-205. 
11. Minakov, A.V., Platonov, D.V., Dekterev, A.A., Sentyabov, A.V., Zakharov, A.V., 2015, The analysis of unsteady flow structure and low frequency pressure pulsations in the highhead Francis turbines. Int. J. Heat and Fluid Flow 53: 183-194.

12. Smirnov, R., Shi, S., Celik, I., 2001, Random flow generation technique for large-eddy simulations and particle-dynamics modeling, J. Fluids Engineering. 123: 359-371.

13. Shtork S.I., Cala C.E., Fernandes E.C., 2007, Experimental characterization of rotating flow field in a model vortex burner, Exp. Therm. Fluid Sci. 31: 779-788.

14. Nicoud, F. and Ducros, F., 1999, Subgrid-Scale Stress Modelling Based on the Square of the Velocity Gradient Tensor. Flow, Turbulence and Combustion, 62(3). 183-200.

15. Picano, S. and Hanjalić, K., 2012, Leray- $\alpha$ regularization of the Smagorinsky-closed filtered equations for turbulent jets at high Reynolds numbers, Flow, Turbulence and Combustion, 89: 627-650.

16. Skripkin, D.G., Tsoy, MA. Shtork, S.I. and Hanjalic, K. Comparative analysis of twin vortex ropes in laboratory models if two hydroturbine draft tubes. (under consideration for publication in J. Hydraulic Research).

17. Minakov, A., Platonov, D., Litvinov, I., and Hanjalić, K., 2015, Computer simulation of flow at part load in a laboratory model of a Kaplan hydroturbine by RANS, DES and LES. In: Hanjalić et al (Eds), Turbulence, Heat and Mass Transfer 8, Begell House Inc., New York, 849-853. 